\title{
Evaluation of a cognitive psychophysiological model for management of tic disorders: an open trial
}

\author{
Kieron O'Connor, Marc Lavoie, Pierre Blanchet and Marie-Ève St-Pierre-Delorme
}

\section{Background}

Tic disorders, in particular chronic tic disorder and Tourette syndrome, affect about $1 \%$ of the population. The current treatment of choice is pharmacological or behavioural, addressing tics or the premonitory urges preceding tic onset.

\author{
Aims \\ The current study reports an open trial evaluating the \\ effectiveness of a cognitive psychophysiological treatment \\ addressing Tourette-specific sensorimotor activation \\ processes rather than the tic.
}

\section{Method}

Forty-nine people with Tourette syndrome and 36 people with chronic tics completed 10 weeks of individual cognitive psychophysiological therapy. Outcome measures included two tic severity scales and psychosocial measures.

\section{Results}

Post-treatment both groups had significantly improved on the tic scales with strong effect sizes across tic locations and complex and simple tics, maintained at 6-month follow-up with further change in perfectionism and self-esteem.

\section{Conclusions}

The cognitive psychophysiological approach targeting underlying sensorimotor processes rather than tics in Tourette's and chronic tic disorder reduced symptoms with a large effect size.

\section{Declaration of interest}

K.O'C. receives royalties from Wiley, Elsevier and MultiMonde publishers for published work. P.B. receives an honorarium from UCB Pharma Canada and speaker fees from Novartis Pharma Canada.

\section{Copyright and usage}

(c) The Royal College of Psychiatrists 2016.
Tics are defined as repetitive non-voluntary contractions of functionally related groups of skeletal muscles in one or more parts of the body. Tics occur across all cultures and have been reported anecdotally since classical times. Currently, the Diagnostic Statistical Manual of the American Psychological Association (DSM-APA) distinguishes chronic tic disorders and Gilles de la Tourette syndrome. Tourette syndrome (or disorder) is recognised in the DSM-IV and 5 as a separate diagnostic category with multiple tics including vocal (phonic) tics occurring several times per day, for at least 1 year with onset before age 18 . Tics may be simple or complex. Simple tics include blinking, cheek twitches, head or knee jerks and shoulder shrugs. Complex tics may involve sequences of movements, and may be bizarre mannerisms involving limbs, head or extremities. Tics are mainly confined to the upper body and the most common occur in the eye, head, shoulders and face. Vocal tics include coughs, tongue clacking, sniffing, throat clearing, hiccing, barking and growling.

Tics are usually preceded by discomforting bodily sensations, known as sensory phenomena, which provide an involuntary urge to tic. ${ }^{1}$ Sensory phenomena include feelings of discomfort, incompleteness, pressure, tension, tingling, warmth, increased energy or even an attribution and are correlated with tic severity. ${ }^{2,3}$ Tourette syndrome is commonly diagnosed around the age of 6 years and follows a waxing and waning course of tic severity, intensity and frequency. ${ }^{4}$ Estimates have placed the prevalence of Tourette syndrome as high as $1 \%$ in adults and $3 \%$ in children, and chronic tic disorders at $>10 \%$ of the population. ${ }^{5}$ The ratio of males to females is approximately $4: 1$ and tics peak around age 11 and stay stable or decline in adulthood, although whether tics disappear or adapt in adults remains controversial. ${ }^{6}$

\section{Current treatments}

Current pharmacological treatment options include drugs that lower or block the action of dopamine including typical ${ }^{7}$ and atypical $^{8}$ antipsychotics which improve Tourette syndrome symptoms. Current Canadian and European guidelines, while recognising pharmacotherapy, consider behavioural interventions as the treatment of choice. ${ }^{9,10}$

The treatments of choice for managing the tics include habit reversal training (HRT). ${ }^{11}$ Comprehensive Behavioural Intervention for Tics (CBIT) is an updated version of habit reversal whose key component is rehearsal of a competing response to the tic, and one large-scale multisite study comparing CBIT with support therapy found a significantly greater decrease in tics following CBIT in children ${ }^{12}$ as well as in adults. ${ }^{13}$ Previous studies applying CBIT to tic disorders have reported effect sizes (standardised mean differences in post-test) between 0.55 and 0.63 for adults and 0.68 for children ${ }^{12}$ compared with active control. A comparable behavioural treatment for managing tics may be exposure and response prevention (ERP) which has been shown as effective as HRT in reducing tic frequency, ${ }^{14}$ although duration of treatment differed across modality. ${ }^{15}$

The cognitive psychophysiological model of tic behaviour presents an alternative cognitive-behavioural approach to ERP and HRT (CBIT). Rather than addressing the tic in isolation, the cognitive psychophysiological intervention aims to change the background activity against which the tic occurs. The cognitive psychophysiological model is based on experimental findings that people with tic disorders show problems planning optimally and in particular inhibiting responses when the tasks involve automatic planning. Other motor faculties involving motor speed, proprioceptive feedback and guided aiming movements seem 
intact. ${ }^{16}$ The difficulty in planning and inhibiting movements seems to manifest itself in everyday life through overactivity and overpreparation in planning action as measured by a style of planning action (STOP) questionnaire and manifested in a high level of sensorimotor activation. ${ }^{17}$ Hence a key factor in the cognitive psychophysiological intervention is to modify planning and inhibiting motor action, centrally and peripherally, so preventing tic onset.

Although these problems in planning seem to be organisational, they are not exclusively linked to executive function, hyperactivity and impulsivity. The STOP subscales measuring everyday overactivity relate rather more to perfectionism than to impulsivity. ${ }^{18}$ The cognitive psychophysiological approach involves also (meta)cognitive identification of how the person evaluates high-risk compared with low-risk situations/activities and exploring how the planning style fits with pre-existing perfectionist beliefs.

An initial randomised (3:1) waitlist controlled trial incorporating some aspects of the cognitive psychophysiological programme into habit reversal demonstrated its efficacy on 47 individuals with mild-moderate chronic tic disorder and 43 individuals with body focused repetitive impulse disorders (e.g. hair pulling, nail biting, teeth grinding) receiving a 4-month treatment programme. ${ }^{19}$ Furthermore, normalisation of motor performance and electrocortical motor potentials changes were correlated with symptom improvement, further validating the cognitive psychophysiological model. ${ }^{20}$

The aim of the present study was to further validate the cognitive psychophysiological programme of addressing tic disorders via targeting Tourette-specific planning processes and accompanying sensorimotor activation (rather than the isolated tics themselves) through a combination of cognitive-behavioural and psychophysiological interventions.

The reasons for conducting an open trial of the cognitive psychophysiological programme prior to a controlled randomised trial was to evaluate the efficacy of the programme over a range of tics including simple, complex, vocal, motor and tic locations with all degrees of severity (chronic tic disorders and Tourette syndrome). Since steps in the programme were additive, a secondary aim examined whether the process of change where it occurred was uniform and cumulative over tic samples.

\section{Method}

\section{Recruitment}

Recruitment was via announcements and journalist reporting addressed to the general public. Consecutive referrals were screened and evaluated by a two-stage process. Initially they were screened by telephone for suitability and authenticity, then, they were invited to attend a semi-structured interview where they also completed questionnaires, plus a semi-structured evaluation.

Criteria for inclusion were: presenting a simple/complex tic for at least 1 year occurring daily. Those included in the Tourette syndrome group, ages 18-65, had a diagnosis of Tourette syndrome as the principal presenting problem accompanied by vocal tics. Those included in the chronic tic disorder group presented a simple/complex tic (vocal or motor) for at least 1 year occurring daily, and were French or English speaking. Criteria for exclusion were: any major medical history, head injury including sensorimotor impairment, history of autism, IQ $<75$; other psychiatric problem on Axis I or II requiring treatment (minor comorbidities were accepted); any neurological problems (e.g. Parkinson's, hemifacial spasms, Meige syndrome, sclerosis; Huntington's disease, Wilson's disease); currently receiving treatment from a psychologist, acupuncturist, hypnotherapist, massotherapist; currently receiving psychotropic drugs non-relevant to Tourette syndrome or attention-deficit hyperactivity disorder (ADHD), or misuse of alcohol or drugs.

Subsequent to passing the telephone screening, an appointment was made with the neurologist collaborating on the project (P.B.) for neurological screening. An independent clinical psychologist then administered the Tourette Syndrome Global Scale (TSGS) ${ }^{21}$ to assess participants on tic disorder and severity of Tourette syndrome symptom rating, and a subset received also the Yale Global Tic Severity Scale (YGTSS). ${ }^{22}$ The evaluators were masked to the treatment and hypotheses. After confirmation that the person met the inclusion criteria for the study (and none of the exclusion criteria), the participant received a psychological assessment. The psychological assessment included the Obsessive-Compulsive-Tourette Scale ${ }^{23}$ (OC-TS; a semistructured interview) and aimed to uncover in more detail the person's tic problem, history, current situation, motivation and degree of disruption of everyday life by the tic and other factors surrounding the problem.

\section{Participants}

At the start of therapy, the two groups included 67 individuals with Tourette syndrome and 62 individuals with chronic tic disorders. Eleven people in the Tourette syndrome group and 16 people in the chronic tic disorders group refused treatment, leaving 56 and 46 participants respectively (subsequently 7 individuals with Tourette syndrome and 10 individuals with tic disorders abandoned treatment before completion). The total sample in each group was: Tourette syndrome (pre $=56$; post $=49$; follow-up $=25$ ); chronic tic disorders ( $\mathrm{pre}=46$; post $=36$; followup =27). All participants read and gave witnessed signed informed consent and the project was approved by the local institutional ethics board. The consort flow chart is given in Fig. 1.

\section{Clinical assessment}

Axis I disorders were assessed with the Structured Clinical Interview for DSM-IV (SCID-I). ${ }^{24}$ Axis II personality disorders were screened with the Personality Disorder Questionnaire $(\mathrm{PDQ}-4)^{25}$ prior to assessment. Assessment of ADHD followed current guidelines ${ }^{26}$ and included ADHD rating scales, a diagnostic interview and symptom-specific measures.

Tic disorder symptom severity was assessed principally with the TSGS $^{21}$ but the Yale Global Tourette Syndrome Scale (YGTSS) $)^{22}$ was administered to a subset of participants. Both questionnaires were administered by the clinician via a structured interview. The TSGS is the older tic symptom rating scale and was preferred over the YGTSS due to its multidimensional nature and increased sensitivity of its scoring to multidimensional change. The TSGS norms also allow classification into mild, moderate and severe symptoms. The first TSGS subscale rates the nature of the tic (i.e. motor or phonic), whereas the second scale rates the tic complexity. A third scale assesses functional impairment, including behavioural, learning, motor restlessness and occupational problems. According to past research, ${ }^{22}$ the interrater reliability of the TSGS global score was found to be very good $(k=0.77, P<0.001)$. The YGTSS is also a clinician-rated scale used to assess tic severity and impairment due to tics. ${ }^{22}$ Motor and vocal tics are rated separately from 0 to 5 on distinct dimensions (number, frequency, intensity, complexity and interference). The scale yields a total score for motor tics (0-25), a total score for vocal tics $(0-25)$ and a combined total tic score $(0-50)$. Convergent validity of the motor and phonic tic factors is shown 


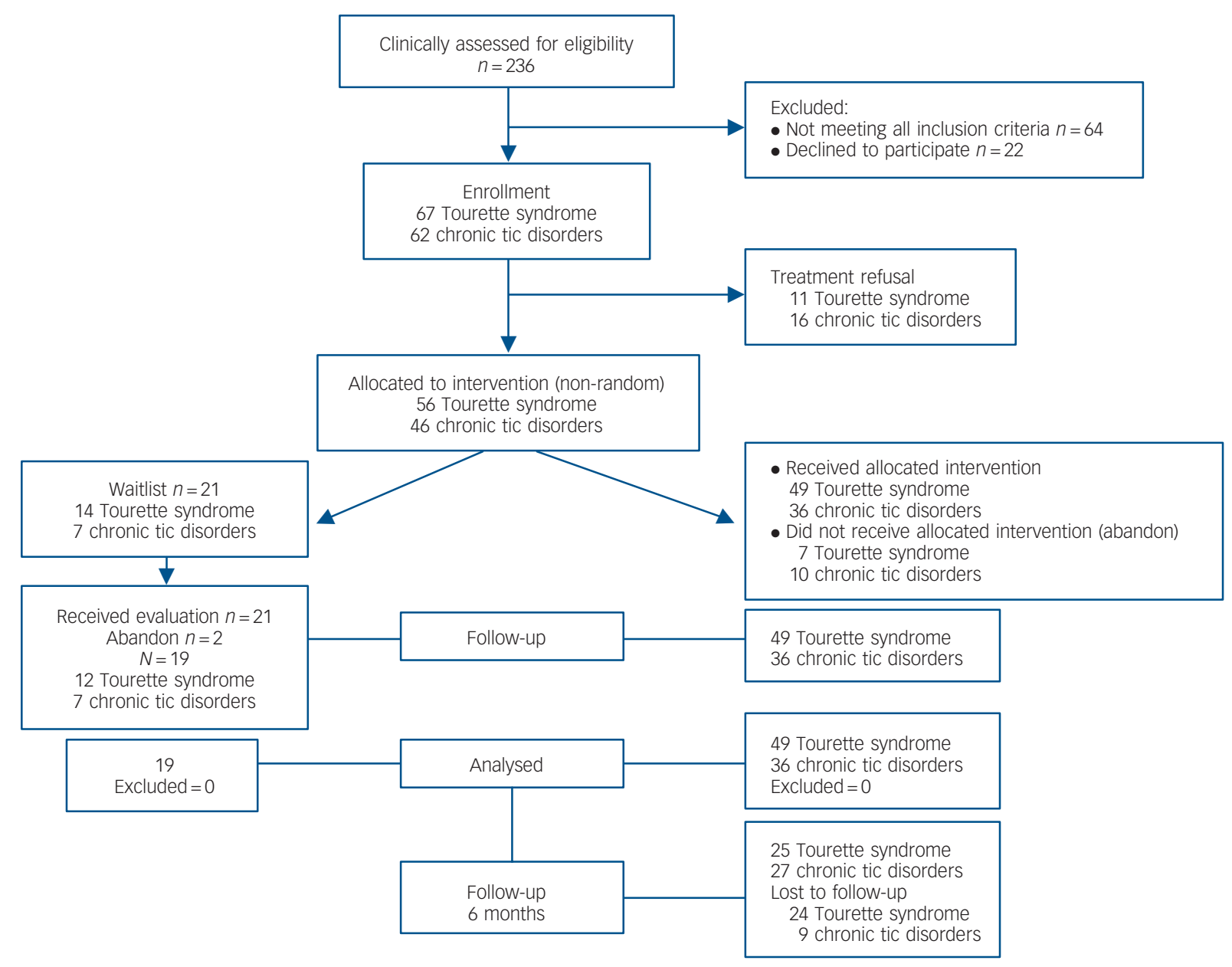

Fig. 1 Consort flow chart.

by strong correlations between the TSGS and the YGTSS, ranging from $r=0.86$ to $r=0.91 .^{22}$ The YGTSS was given to a random percentage of participants ( 24 with chronic tic disorders, 15 with Tourette syndrome) as a check on TSGS evaluation to give some indication of convergence between two measures. Participants were also assessed on the OC-TS semi-structured interview, which poses questions on history, onset, form, location and nature of the tic and the presence, nature and duration of any premonitory sensory urge (OC-TS). After this clinical assessment, the participant was asked to read and, if they were willing, to sign a consent form to enter the neurocognitive evaluation phase.

The SCID-I, TSGS, YGTSS and OC-TS evaluations were carried out by trained evaluators (psychologists, doctoral students) who had previously received training in administering the semi-structured interviews. Further, they did not receive other than basic demographic information prior to their evaluation. As far as possible, the same evaluator evaluated the participant pre and post intervention. Six-month follow-up was also conducted by an independent evaluator.

\section{Medication}

Patients with Tourette syndrome who were currently under medication and stabilised were also included. Stabilisation on medication implied receiving typical or atypical antipsychotic medication or antidepressant or psychostimulant medication over at least a 3-month period with no further improvement in symptoms and willing to keep dosage constant over the period of the study. Medications seem not to influence CBIT outcome. ${ }^{27}$ Table 1 lists participant medications.

\section{Waitlist}

A natural waitlist control group consisted of 21 participants who completed clinical assessment but were not treated (for a variety of natural reasons, e.g. availability, wait for therapist, absence) before 3 months when 19 were clinically reassessed (12 Tourette syndrome, 7 chronic tic disorders, $57 \%$ male). A planned waitlist is prohibited by Canadian tri-council policy where an empirically based effective treatment already exists.

\section{Refusers, withdrawals and completers}

Participants were considered treatment refusals if they never progressed to full evaluation for entry criteria. Completers were defined as those completing post-treatment evaluation, and withdrawals were defined as those voluntarily or involuntarily stopping therapy after evaluation and before completion.

\section{Diagnosis and treatment integrity}

Treatment diagnosis was checked by an independent trained evaluator on whether she concurred with classification of the patient as having Tourette, chronic tic or other disorder. The diagnosis was checked on a random sample of $30(25 \%)$ audiotapes. Similarly, treatment integrity and delivery were assessed by rating the presence of each stage of therapy in the treatment notes. 


\section{Questionnaire measures}

The Beck Anxiety Inventory $(\mathrm{BAI})^{28}$ was administered and consists of a 21-item anxiety symptom checklist rating symptom intensity for the past week on a $0-3$ scale. Its total score ranges from 0 to 63. The French version of the BAI shows good psychometric properties (internal consistency, test-retest stability, convergent and divergent validity). ${ }^{29}$

The Beck Depression Inventory (BDI-II $)^{30}$ consists of a 21 -item relative to depression. The BDI assesses cognitive, emotional and somatic depressive symptoms on a $0-3$ scale. Its total score ranges from 0 to 63 . The French version of the BDI shows good psychometric properties (internal consistency and test-retest reliability). ${ }^{31}$

The Frost Multidimentional Perfectionism Scale (MPS) ${ }^{32}$ is a self-administered questionnaire of 35 items $(0-5)$ covering six dimensions ( 1 - concern over mistakes; 2 - personal standards; 3 - parental expectations; 4 - parental criticism; 5 - doubts about actions; and 6 - importance of organisation and order). The coefficient of internal consistency in both English and French is satisfactory with good test-retest reliability. Total score ranges from 35 to 175 .

The Barratt Impulsiveness Scale (BIS-10) ${ }^{34}$ is one of the oldest and most widely used measures of impulsive personality traits on a 4-point scale (1-4). It includes 34 items measuring attention, motor, impulsiveness and non-planned impulsiveness. A total score ranges from 34 to 136 . The internal consistency coefficient for the total score is good. The scale has been validated in French. ${ }^{35}$

The Padua Inventory (PI $)^{36}$ assesses obsessive-compulsive behaviour. The psychometric properties of the French version of the PI are adequate (test-retest validity, convergent and divergent validity). ${ }^{37} \mathrm{~A}$ revised version of the PI was used for the current study, ${ }^{38}$ with subscales representing impulse phobia, washing, checking, rumination and precision symptoms. On this revised version, the score ranges from 1 to 5 .

The Social Self-Esteem Inventory $(\mathrm{SSEI})^{39}$ is a 30 -item measure of the self-esteem which shows internal consistency $(\alpha=0.93)$ and test-retest reliability $(r=0.95) .{ }^{40}$ Scores range from 30 to 180 .

\section{Credibility, expectancy, motivation and therapist evaluation}

Credibility, expectancy and motivation were derived from the Devilly-Borkovec credibility/expectancy questionnaire. ${ }^{41}$ This questionnaire consists of a total set of six items measuring credibility (feeling) and expectancy (thinking) on two scales rated from 1 to 9 on each item, except two items $(0-100)$ for a total score of 236. Cronbach's alpha is respectively 0.79 and 0.81 for each scale, with high test-retest reliability 0.83 . The expectancy scale correlates with outcome changes $(r=0.74)$. The adapted motivation questionnaire ${ }^{42}$ contains 10 items (5 reversed) and focuses on reasons to change tic behaviour and willingness to change for a total score of 10 .

\section{Daily diary}

The participants also kept a daily diary at baseline and throughout treatment monitoring frequency, intensity and degree of control in a specially prepared booklet. The participants were trained in the use of the booklet and a unit of tic or habit defined at the beginning of the evaluation. These records were computerised and displayed as graphics mainly to give clinical feedback to the participant. However, an average of 14 completed daily diaries (7 chronic tic disorders, 7 Tourette syndrome) was chosen randomly from the completers to calculate the linearity of progress over therapy components.

\section{Treatment protocol}

The cognitive psychophysiological treatment comprises 10 cumulative stages administered over 10 sessions: (a) awareness exercises (daily diary, video); (b) muscle discrimination (normalise contractions, gain in tic-affected muscle flexibility; exercises are independent of tic occurrence; (c) prevention of tension through decontraction; (d) identifying activities at high and low risk for tics; (e) highlighting differences in behaviours, thoughts and feelings and approach associated with high- and low-risk activities; (f) identifying STOP producing tension (overpreparation, overactivity, inflexibility); (g) behavioural restructuring of overpreparation and overactive style of action; (h) cognitive-behavioural restructuring of metacognitive and perfectionist beliefs linked to tension; (i) generalisation; and (j) relapse prevention. So the treatment sequence for one individual at high risk for shoulder tics while driving was to increase flexibility in the tic-affected muscles and normalise the contraction appropriate for gripping a steering wheel. Perfectionist beliefs about intensely staring into ongoing traffic were restructured and the individuals' agenda reorganised to leave more time and less rush between travel appointments. As noted, the programme is based on a series of findings suggesting that people with Tourette syndrome experience perfectionist beliefs when planning action, feeding an impulsive overactive style which then feeds frustration, tension and tics. Tics are not directly addressed either by the practice of antagonist competing response contingency management and positive reinforcement of competing responses or exposure to the premonitory urge.

Each individual, whether in the Tourette syndrome or chronic tic disorders group, received the identical 10 stages during 10-week therapy, with 1-month home practice and 6 months of follow-up. All therapists were licensed psychologists with between 5 and 7 years of experience specialising in cognitive-behavioural therapy for tic disorders.

\section{Analysis}

An initial multivariate analysis of variance was carried out to look at pre-post differences in main clinical outcome measures (TSGS, YTSS) and other mood-related and psychosocial measures (BIS-10, BAI, BDI, SSEI, PI) in both completers and in an intention to treat (ITT) analysis. In the ITT, scores at end-point of abandon were carried forward to represent final scores (end-point carried forward). A further analysis was performed post 6-month follow-up on completers to look at relapse from post-treatment levels. Finally, outcome was compared according to baseline classifications of TSGS tic severity.

\section{Results}

\section{Demographic differences between completers and those who dropped out}

Baseline demographic data are given in Table 2. Differences were found on age: completers were older $(\bar{x}=41.05$; s.d. $=12.84)$ compared with those who dropped out $(\bar{x}=35.9$; s.d. $=5.93)$; more educated (chi-squared $=8.69 ; P<0.03$ ); and more likely to be married (chi-squared $=18.38 ; P<0.003$ ). But there were no differences in level of symptom severity as measured by the TSGS, either between dropouts and completers. There were no demographic differences between completers and the natural waitlist control group.

\section{Clinical measures}

Multivariate repeated measures on the two groups pre- and post-treatment revealed for the chronic tic disorders group a 


\begin{tabular}{|c|c|c|c|c|}
\hline \multirow[b]{2}{*}{ Demographics } & \multicolumn{2}{|c|}{ Chronic tic disorders } & \multicolumn{2}{|c|}{ Tourette syndrome } \\
\hline & Frequency & s.d. & Frequency & $\%$ \\
\hline Age, years: mean (s.d.) & \multicolumn{2}{|c|}{$41.87 \quad(1.69)$} & \multicolumn{2}{|c|}{$38.08 \quad(1.56)$} \\
\hline \multicolumn{5}{|l|}{ Education level } \\
\hline Primary school & 0 & 0 & 5 & 8.93 \\
\hline High school & 11 & 24.44 & 16 & 28.57 \\
\hline Vocational college & 11 & 24.44 & 12 & 21.43 \\
\hline University & 23 & 51.11 & 23 & 41.07 \\
\hline \multicolumn{5}{|l|}{ Civil status } \\
\hline Single & 12 & 32.69 & 16 & 29.09 \\
\hline Married or cohabitation & 26 & 56.52 & 33 & 60.00 \\
\hline Divorced or separated & 7 & 15.22 & 6 & 10.91 \\
\hline Widowed & 1 & 2.17 & 0 & 0 \\
\hline \multicolumn{5}{|l|}{ Gender } \\
\hline Female & 19 & 41.30 & 24 & 43.64 \\
\hline Male & 27 & 58.69 & 32 & 58.18 \\
\hline \multicolumn{5}{|l|}{ Comorbidity } \\
\hline Attention-deficit hyperactivity disorder & 3 & & 0 & \\
\hline \multicolumn{5}{|l|}{ Medication } \\
\hline Antidepressive & 6 & 13.04 & 13 & 23.21 \\
\hline Anxiolytic & 0 & 0 & 3 & 5.36 \\
\hline Other & 6 & 13.04 & 8 & 14.29 \\
\hline Non-medicated & 34 & 73.91 & 32 & 57.14 \\
\hline
\end{tabular}

\begin{tabular}{|c|c|c|c|c|c|c|}
\hline \multirow[b]{2}{*}{ Measures } & \multicolumn{3}{|c|}{ Chronic tic disorders } & \multicolumn{3}{|c|}{ Tourette syndrome } \\
\hline & N & Pre, mean s.d. & Post, mean s.d. & $n$ & Pre, mean s.d. & Post, mean s.d. \\
\hline \multicolumn{7}{|c|}{ Intention to treat analysis } \\
\hline TSGS total & 46 & $25.53(12.34)$ & $10.95(4.86)$ & 56 & $18.05(11.13)$ & $9.52(9.86)$ \\
\hline Tics & 46 & $12.03(9.73)$ & 4.90 (4.73) & 56 & $10.33(8.89)$ & $5.22(5.41)$ \\
\hline Behaviour & 46 & $13.51(7.60)$ & $6.06(5.50)$ & 56 & $7.72(4.84)$ & $4.30(3.61)$ \\
\hline YGTSS (50) & 44 & $17.02(6.43)$ & $14.16(5.56)$ & 24 & $24.91(7.44)$ & $20.08(9.33)$ \\
\hline \multicolumn{7}{|c|}{ Completer analysis } \\
\hline TSGS total & 36 & $19.15(11.76)$ & $9.65(7.97)$ & 49 & $22.78(12.45)$ & $10.48(8.06)$ \\
\hline Tics & 36 & $8.45(5.26)$ & 4.46 (3.73) & 49 & $12.60(6.76)$ & $5.85(5.41)$ \\
\hline Behaviour & 36 & $10.89(8.43)$ & $5.25(5.43)$ & 49 & $10.58(7.93)$ & $4.60(4.73)$ \\
\hline YGTSS (50) & 24 & $16.75(6.18)$ & $11.50(5.56)$ & 15 & $23.33(6.16)$ & $15.60(6.32)$ \\
\hline BAl & 36 & $7.16(5.22)$ & $4.67(5.28)$ & 49 & $11.58(10.27)$ & $6.95(6.42)$ \\
\hline $\mathrm{BDI}$ & 36 & $10.79(10.2)$ & $7.31(8.69)$ & 49 & $9.21(9.05)$ & 4.18 (5.3) \\
\hline $\mathrm{Pl}$ & 36 & $25.00(19.8)$ & $26.00(15.9)$ & 49 & $29.87(19.9)$ & $26.98(18.1)$ \\
\hline MPS & 36 & $103.57(22.55)$ & $95.72(18.65)$ & 49 & $93.55(24.59)$ & $86.32(23.95)$ \\
\hline BIS-10 & 36 & $72.96(10.72)$ & $76.21(11.02)$ & 49 & $68.26(15.95)$ & $74.88(12.55)$ \\
\hline SSEI & 36 & $129.41(30.88)$ & $128.09(31.93)$ & 49 & $127.63(26.12)$ & $129.34(26.18)$ \\
\hline
\end{tabular}

significant main treatment effect for the total TSGS scale $\left(F[1,35]=25.86 ; \quad P<0.001 ; \quad d^{-}=1.72\right) \quad(95 \% \quad$ CI $\quad$ pre $=15.35-$ 22.94; post $=7.16-12.15)$; and for the separate tic subscale $\left(F[1,35]=20.2 ; \quad P<0.001 ; d^{-}=1.52\right)$ and behavioural subscale $\left(F[1,35]=19.01 ; P<0.0001 ; d^{-}=1.48\right)$ and on the YGTSS total score $\left(F[1,23]=30.93 ; P=0.001 ; d^{-}=2.36\right)$ : tic disorders $(95 \%$ CI pre $=14.72-18.78$; post $=0.35-13.65$ ).

In the Tourette syndrome group the results pre- and post-treatment were for the total TSGS scale $(F[1,48]=82.72$; $\left.P<0.0001 ; d^{-}=2.63\right) \quad(95 \%$ CI pre $=19.52-22.94$; post $=8.34-$ $12.62)$, for the separate TSGS tic scale $(F[1,48]=74.1$; $\left.P>0.0001 ; d^{-}=2.49\right)$, TSGS behavioural scale $(F[1,48]=43.24$; $\left.P<0.001 ; d^{-}=1.89\right)$ and on the YGTSS (50) total score $\left(F[1,14]=27.61 ; P<0.001 ; d^{-}=2.81\right)$ : Tourette syndrome $(95 \%$ CI pre $=20.76-25.90$; post $=12.88-18.32$ ). ITT analysis using the end-point carry forward method confirmed the significant findings pre- and post-treatment on TSGS for combined chronic tic disorders and Tourette syndrome groups $(F[1,112]=78.39$; $\left.P<0.001 ; d^{-}=1.40\right)$. There were no differences in outcome between participants with simple and complex tics $(P>0.11)$ or across tic locations $(P>0.08)$. Tic locations were analysed separately. The tics were classified as follows: simple tics $(n=49)$ $d=1.17$; complex tics $(n=36) d=0.99$; eyes $(n=10) d=1.03$; face $(n=6) \quad d^{-}=1.06$; head $(n=7) d^{-}=0.95$; neck and shoulder $(n=17) d^{-}=1.61$; trunk and abdomen $(n=9) d=1.14$; phonic $(n=11) d=1.16$, for individual tic locations.

There were no differences in outcome on the TSGS between genders $(P<0.14)$ or between those taking or not taking medication (chronic tic disorders, $P<0.70$; Tourette syndrome, $P<0.37)$. The YTGSS and TSGS total scores pre-treatment 
showed a highly significant positive correlation $(F[38]=0.45$; $P<0.0001)$. Clinical and questionnaire data are given in Table 2 .

\section{Clinical significance}

In terms of clinically significant reductions, in the chronic tic disorders group, $64.7 \%$ and in the Tourette syndrome group, $73.9 \%$ showed significant $(<35 \%)$ improvement in symptoms on the TSGS post-treatment which is considered a full response to treatment. ${ }^{43}$ Completers with baseline ratings on the TSGS between mild $(>10)$, moderate $(>20)$ and severe $(>30)$ as categorised by TSGS norms improved equally post-therapy. The odds of clinical improvement depending on initial mild, severe, moderate category of TSGS total score were equal in both groups. In the chronic tic disorders group, $64 \%$ of mild and $67 \%$ of moderate-severe showed $>35 \%$ improvement (odds ratio $(\mathrm{OR})=1.1: 1)$. In the Tourette syndrome group, $71 \%$ with mild Tourette syndrome and $79 \%$ with moderate-severe syndrome showed improvement $(\mathrm{OR}=1.5: 1)$. The likelihood of improving with the treatment compared with waitlist was $6: 1$ in the total sample (Tourette syndrome and chronic tic disorders). At posttherapy, of 85 completers, 78 were classified as mild or below, regardless of starting severity (mild, moderate, severe, extreme) and the other 7 were classified as moderate.

\section{Waitlist results}

The waitlist was small but there were no demographic differences in this group, compared with treatment groups. The TSGS total score showed a slight decrease $(\operatorname{pre}(19)=19.62 \quad(3.81)$, $\operatorname{post}(19)=17.05(2.67))$. This post-waitlist decrease was nonsignificant over the 19 participants (tic disorders+Tourette syndrome) allocated to the waitlist $(F[1,18]=0.80 ; P>0.38$, $\left.d^{-}=0.29\right)$.

\section{Questionnaire measures}

In the tic disorders group, the BAI showed a decrease pre-post treatment $\left(F[1,35]=3.79 ; P<0.006 ; d^{-}=0.68\right)$ with no interaction effect. But BAI levels were subclinical at baseline. The BDI-II also showed a decrease $\left(F[1,35]=7.95 ; P<0.008 ; d^{-}=0.92\right)$. BDI-II levels were again subclinical. The PI total showed no decrease pre-post treatment. The BIS-10 questionnaire showed a trend in groups pre-post treatment. The SSEI showed no change. The MPS showed a significant pre-post treatment effect $\left(F[1,35]=7.81 ; P<0.001 ; d^{-}=1.06\right)$.

The Tourette syndrome group, likewise, showed improvement in depression and anxiety: BDI-II $(F[1,48]=15.01 ; P<0.001$; $\left.d^{-}=1.67\right) ; \quad$ BAI $\quad\left(F[1,48]=6.79 ; \quad P<0.013 ; \quad d^{-}=0.88\right) ; \quad$ MPS
$\left(F\left[1,30=5.45 ; P<0.03 ; d^{-}=0.86\right) ;\right.$ and the BIS-10 $(F[1,23]=$ 6.56; $P<0.02)$. But there was no significant change in PI or SSEI.

\section{Six-month follow-up}

A 6-month follow-up with clinical assessment was conducted on completers showing improvement post-treatment. At 6-month follow-up, there was no relapse in the TSGS (chronic tic disorders $\bar{x}=7.47$ [6.8]; Tourette syndrome $\bar{x}=11.91$ [8.2]). In the chronic tic disorders group there was a further decrease in BAI $\left(F[1,20]=3.94 ; \quad P<0.06 ; \quad d^{-}=0.89\right) \quad$ and $\quad$ in total MPS $\left(F[1,17]=7.46 ; P<0.01 ; d^{-}=1.32\right)$. In the Tourette syndrome group there was an increase of SSEI from post-level $(F[-1,18]=$ 7.96; $\left.P<0.01 ; d^{-}=1.33\right)$ and in total MPS $(F[1,28]=7.81$; $\left.P<0.001 ; d^{-}=1.06\right)$, but in no other measure. In both the chronic tic disorders and Tourette syndrome group, there was further change at follow-up on the MPS on the personal standards scale (chronic tic disorders: $F[1,17]=6.17 ; P<0.02 ; d^{-}=1.20$; Tourette syndrome: $\left.F[1,17]=4.48 ; P<0.05 ; d^{-}=1.02\right)$ and in the Tourette syndrome group on personal organisation $(F[1,17]=5.74$; $\left.P<0.03 ; d^{-}=1.16\right)$. Pearson correlation coefficients were calculated between change in the MPS and change in the TSGS scales at follow-up. Change in the personal organisation subscale of the MPS correlated significantly with the change in TSGS behaviour scale post-treatment $(r[85]=0.31 ; P<0.02)$ and at 6-month follow-up with change in the TSGS behaviour scale $(r[27]=0.39 ; P<0.05)$ and the TSGS tic scale $(r[27]=0.40$; $P<0.04)$, and the TSGS total score $(r[27]=0.49 ; P<0.009)$.

\section{Treatment motivation, credibility, adherence and satisfaction}

The groups were equivalent post-treatment in terms of motivation (chronic tic disorders [46] $=7.38$ [0.98]; Tourette syndrome $[56]=7.10$ [1.57]); combined credibility and feeling of therapy pre-treatment (chronic tic disorders [46] pre $=157.70$ [32.57]; Tourette syndrome [56] pre $=162.41$ [30.22]); and adherence to programme as monitored by attendance, homework completion and exercise practice as computed mid- and post-treatment by therapist (chronic tic disorders [15] 6.32/12 [2.58], Tourette syndrome [17] 7.05/12 [3.28] and close other post-treatment $(0=$ not at all; $2=$ diligently; Tourette syndrome $[11]=1.55$ [0.52]; tic disorders $[12]=1.33[0.49])$.

\section{Evolution of progress during therapy}

Fourteen case samples from each group (7 tic disorders and 7 Tourette syndrome) were extracted from the completed daily diary

Table 3 Daily tic unit frequency from daily diary showing mean (standard deviation) averaged over 7 chronic tic disorder and 7 Tourette syndrome completers for each of the 10 weekly stages of the programme

\begin{tabular}{|c|c|c|c|c|}
\hline & \multicolumn{2}{|c|}{ Chronic tic disorder } & \multicolumn{2}{|c|}{ Tourette syndrome } \\
\hline & $\bar{x}$ & s.d. & $\bar{x}$ & s.d. \\
\hline Evaluation & 28.30 & 38.5 & 22.87 & 11.8 \\
\hline Stage 1 & 17.51 & 20.4 & 17.97 & 21.0 \\
\hline Stage 2 & 15.72 & 17.4 & 17.31 & 14.6 \\
\hline Stage 3 & 15.59 & 20.4 & 16.60 & 17.5 \\
\hline Stage 4 & 10.08 & 10.0 & 13.41 & 15.6 \\
\hline Stage 5 & 4.77 & 8.6 & 11.62 & 12.1 \\
\hline Stage 6 & 4.57 & 8.8 & 10.98 & 10.6 \\
\hline Stage 7 & 6.37 & 9.3 & 10.58 & 11.4 \\
\hline Stage 8 & 5.65 & 7.5 & 9.63 & 11.0 \\
\hline Stage 9 & 4.01 & 7.6 & 8.78 & 9.4 \\
\hline Stage 10 & 4.29 & 7.7 & 7.40 & 7.2 \\
\hline
\end{tabular}


to measure the evolution of tic and distress reduction over the 10 sessions of therapy to see whether the reduction followed a linear or curvilinear path in each group. The therapy was administered additively in that all participants received the same stages of therapy in the same order. There was a cumulative and linear improvement over each component of the therapy: frequency $(F[1,13]=6.74 ; P<0.02)$; intensity $(F[1,13]=20.00 ; P<0.001)$; control $(F[1,13]=11.04 ; \quad P<0.006)$; resistance $(F[1,13]=12.23$; $P<0.004)$. The results illustrate that progress in control of tics showed a linear trend in both Tourette syndrome and chronic tic disorders, suggesting that strategies were additive and cumulatively integrated into the learning process (Table 3 ).

\section{Discussion}

The purpose of the open trial was to evaluate the efficacy of the cognitive psychophysiological intervention over a range of tic locations in chronic tic and Tourette disorders. The overall effect size for all groups was robust but in particular the Cohen $d^{-}$effect sizes were large compared with waitlist (relative $d^{-}=2.34$ and 1.43 for Tourette and chronic tic respectively) and large over all tic locations and subtypes (motor, phonic, complex and simple), and cognitive psychophysiological intervention worked effectively in decreasing symptoms regardless of whether baseline TSGS severity was in the mild, moderate or severe category. The results confirm that the cognitive psychophysiological treatment is efficacious for all tic subtypes. Furthermore, it is clear from improvement on both tic and behavioural subscales of the TSGS that not only tics but other behaviour (perfectionism, impulsivity) improved as well as measures of well-being. In the Tourette syndrome group, self-esteem improved at 6-month follow-up.

The cognitive psychophysiological programme targets ways of thinking, feeling and planning, and evaluating motor action characteristic of tic disorders. The cognitive psychophysiological programme, unlike other behavioural interventions, does not directly address the tics themselves, either by encouraging toleration of the premonitory urge or through the habit reversal strategy of implementing an action antagonist to the muscle. The present open trial study shows that the cognitive psychophysiological programme produces equivalent or larger reported effect sizes to other behavioural treatments, as reported in the literature. ${ }^{12,13}$ The cognitive psychophysiological programme may sound more complex than other behavioural interventions in tying tic behaviour to motor planning and style of action, but in practice, it has several advantages. First, in identifying highand low-risk tic situations, it immediately educates people in how they already albeit indirectly have some control over the fluctuations in tic onset. It also directs attention to motor planning processes preceding onset, so permitting the person to realise that reducing the risk of tic onset is often a case of doing less and investing less effort than usual to control tics. In other words, the means of control are often already in the patient's repertoire and cognitive behaviour modification within the cognitive psychophysiological model focuses on normalising thought and action in high-risk tic situations by taking low-risk situations as a point of reference. The cognitive psychophysiological programme does however also contain a component of motor re-education involving improving muscle use and discrimination (gaining muscle flexibility and control) and aiding overactive and inhibitory control. These cognitive and psychophysiological factors may relate to the processes particular to tic production such as high sensorimotor activation, elevated muscle contraction, and so complement application of behavioural principles.

Process measures taken daily over the treatment period revealed that the decrease in tics and increase in control was linear and cumulative over stages of the programme. Each stage was built on the previous stage, improvements in all measures were maintained at 6-month follow-up and perfectionism and selfesteem further improved. The further improvement in social self-esteem at 6-month follow-up is interesting since there was no improvement in those measures post-treatment. Maybe time is required before improvement affects social image. Change in perfectionist personal organisation correlated with change in both behavioural and tic symptom subscales at 6-month follow-up, so confirming a key component of the cognitive psychophysiological model, namely that style of planning and organisation is related to tic onset. Other authors ${ }^{44}$ have suggested that motive in impulse control disorders varies with comorbidity and degree of attribution ${ }^{45}$ and may affect treatment choice.

There are a variety of clinical implications from this study. The importance of the findings is that improvement in tic occurrence can be accomplished by addressing cognitive and motor factors of planning action in tic disorders and not just by targeting antagonist actions in isolated muscles. This route may be more satisfactory for some individuals than directly addressing the tic since, in principle, it aids generalisation. The type and location of a tic in Tourette syndrome are notoriously variable and volatile, and patients with Tourette syndrome talk of experiencing the tic of the day. ${ }^{46}$

The limitations of the present study include it being an open trial, without a substantial placebo and only a small natural waitlist arm. The participants were on a variety of medications and though measures of self-esteem and other traits were measured, wider psychosocial adaptation and functioning were not measured. The drop-out rate was $20 \%$ but there were no differences between completers and those who withdrew on clinical severity. A key reason for drop-out was difficulty scheduling time for appointments and homework perhaps due to an overactive style of action. Future research might use a dismantling design to assess the utility of components of the cognitive psychophysiological programme. It will of course be important to relate the current findings to changes in motor performance pre- and post-treatment to further validate the cognitive psychophysiological process model. Also a randomised controlled comparison of cognitive psychophysiological and CBIT would establish comparable efficacy over subtypes and process and symptom change in adults and children.

\section{Kieron O'Connor, PhD, Marc Lavoie, PhD, Pierre Blanchet, MD, Marie-Ève St-Pierre-Delorme, Research Centre, University Institute of Mental Health at Montreal, Canada \\ Correspondence: Kieron P. O'Connor, Research Centre, University Institute of Mental Health at Montreal, 7331 Hochelaga St., Montreal, Quebec H1N 3V2, Canada. Email: kieron.oconnor@umontreal.ca}

First received 17 Jul 2014, final revision 12 Nov 2014, accepted 2 Dec 2014

\section{Funding}

The study was supported by grant no. 57936 from the Canadian Institute of Health Research and grant no. 20573 from the Fonds de la recherche en santé du Quebec to K.O'C. and M.L.

\section{Acknowledgements}

The authors thank Benoit Deslauriers for statistical assistance.

\section{References}

1 Bliss J. Sensory experiences of Gilles de la Tourette syndrome. Arch Gen Psychiatry 1980; 37: 1343-7.

2 Woods DW, Piacentini J, Himle MB, Chang S. Premonitory urge for tics scale (PUTS): initial psychometric results and examination of the premonitory 
urge phenomenon in youths with tic disorders. J Dev Behav Pediat 2005; 26 397-403.

3 Crossley E, Cavanna AE. Sensory phenomena: clinical correlates and impact on quality of life in adult patients with Tourette syndrome. Psychiatry Res 2013; 209: 705-10.

4 Block MH, Leckman JF. Clinical course of Tourette syndrome. J Psychosom Res 2009; 6: 497-501.

5 Robertson MM, Eapen V, Cavanna AE. The international prevalence, epidemiology, and clinical phenomenology of Tourette syndrome: a cross-cultural perspective. J Psychosom Res 2009; 6: 475-83.

6 Pappert EJ, Goetz CG, Louis ED, Blasucci L, Leurgans S. Objective assessments of longitudinal outcome in Gilles de la Tourette's syndrome. Neurology 2003; 61: 936-40.

7 Shapiro E, Shapiro AK, Fulop G, Hubbard M, Mandeli J, Nordlie J, et al. Controlled study of haloperidol pimozide and placebo for the treatment of Gilles de la Tourette's syndrome. Arch Gen Psychiatry 1989; 46: 722-30.

8 Lombroso PJ, Scahill L, King RA, Lynch KA, Chappell PB, Peterson BS, et al. Risperidone treatment of children and adolescents with chronic tic disorders: a preliminary report. J Am Acad Child AdolesC Psychiatry 1995; 34: 1147-52

9 Steeves T, McKinlay BD, Gorman D, Billinghurst L, Day L, Carroll A, et al. Canadian guidelines for the evidence-based treatment of tic disorders: behavioural therapy, deep brain stimulation, and transcranial magnetic stimulation. Can J Psychiatry 2012; 57: 144-51.

10 Verdellen C, van de Griendt J, Hartmann A, Murphy T. European clinical guidelines for Tourette Syndrome and other tic disorders. Part III: behavioural and psychosocial interventions. Eur Child Adolesc Psychiatry 2011; 20: 197-207.

11 Azrin $\mathrm{HH}$, Peterson AL. Habit reversal for the treatment of Tourette syndrome. Behav Res Ther 1988; 26: 347-51.

12 Piacentini J, Woods DW, Scahill L, Wilhelm S, Peterson AL, Chang S, et al. Behavior therapy for children with Tourette disorder: a randomized controlled trial. JAMA 2010; 303: 1929-37.

13 Wilhelm S, Peterson AL, Piacentini J, Woods DW, Deckersbach T, Sudhodolsky DG, et al. Randomized trial of behavior therapy for adults with Tourette syndrome. Arch Gen Psychiatry 2012; 69: 795-803.

14 Verdellen CWJ, Keijsers GPJ, Cath DC, Hoogduin CAL. Exposure with response prevention versus habit reversal in Tourette's syndrome: a controlled study. Behav Res Ther 2004; 42: 501-11.

15 McGuire JF, Piacentini J, Brennan EA, Lewin AB, Murphy TK, Small BJ, et al. A meta-analysis of behavior therapy for Tourette syndrome. I Psychiatr Res 2014; 50: 106-12.

16 Lavoie ME, Imbriglio TV, Stip E, O'Connor KP. Neurocognitive changes analysis of behavior therapy for Tourette syndrome. J Psychiatr Res 2011; 50 106-12.

17 O'Connor KP. Cognitive Behavioral Management of Tic Disorder. John Wiley \& Sons, 2005.

18 Laverdure A, O'Connor K, Lavoie ME. Cognitive aspects of hyperactivity and overactivity in preadolescents with Tourette syndrome. Psychiatry J 2013; 2013: $7 \mathrm{pp}$.

19 O'Connor KP, Brault M, Loiselle J, Robillard S, Borgeat F, Stip E. Evaluation of a cognitive-behavioral program for the management of chronic tic and habit disorders. Behav Res Ther 2001; 39: 667-81.

20 Lavoie ME, Imbriglio TV, Stip E, O'Connor KP. Neurocognitive changes following cognitive-behavioural treatment in the Tourette's syndrome and chronic tic disorder. Int J Cognit Psychotherapy 2011; 4(Special section: cognitive and neuroscientific approaches to obsessive-compulsive and related phenomena): $34-50$.

21 Harcherik D, Leckman J, Detlor J, Cohen D. A new instrument for clinical studies of Tourette's syndrome. J Am Acad Child Adolesc Psychiatry 1984; 23: $153-60$.

22 Leckman JF, Riddle MA, Hardin MT, Ort SI, Swartz KL, Stevenson J, et al. The Yale Global Tic Severity Scale: initial testing of a clinician-rated scale of tic severity. J Am Acad Child Adolesc Psychiatry 1989; 28: 566-73.

23 Roth RM, Farah C. Questionnaire OC-TS modifié. French version of Yale Child Study Center questionnaire (12/93) (revised in May 2005 by O'Connor K, Pélissier M-C, Laverdure A) [internet]. Montreal, Quebec. Institut universitaire en santé mentale à Montréal, 1999. Available from: http://www.tictactoc.org.
24 First MB, Spitzer RL, Gibbon M, Williams JBW. Structured Clinical Interview for DSM-IV-TR Axis I Disorders, Research Version, Patient Edition (SCID-I/P). Biometrics Research, New York State Psychiatric Institute, 2002.

25 Hyler SE. Personality Questionnaire (PDQ-4). New York State Psychiatric Institute, 1994.

26 Conners CK, March JS, Frances A, Wells KC, Ross R. Treatment of attention deficit/hyperactivity disorder: expert consensus guidelines. J Atten Disord 2001; 4: 7-128.

27 O'Connor KP, Laverdure A, Taillon A, Stip E, Borgeat F, Lavoie ME. Cognitive behavioral management of Tourette's syndrome and chronic tic disorder in medicated and unmedicated samples. Behav Res Ther 2009; 47: 1090-5.

28 Beck AT, Epstein N, Brown G, Steer RA. An inventory for measuring clinical anxiety: psychometric properties. J Consult Clin Psychol 1988; 56: 893-7.

29 Freeston MH, Ladouceur R, Thibodeau N, Gagnon F, Rhéaume J. L'inventaire d'anxiété de Beck: Propriétés psychométriques d'une traduction française [Beck Anxiety Inventory: psychometric properties of a French translation]. L'Encéphale 1994; 20: 47-55.

30 Beck AT, Ward $\mathrm{CH}$, Mendelson $\mathrm{M}$, Mock J, Erbaugh J. An inventory for measuring depression. Arch Gen Psychiatry 1961; 4: 561-71.

31 Bourque $P$, Beaudette D. Étude psychométrique du questionnaire de dépression de Beck auprès d'un échantillon d'étudiants universitaires francophones [Psychometric study of the Beck depression questionnaire in a French-speaking university student sample]. Can J Behav Sci 1982; 14: 211-8.

32 Frost RO, Marten $\mathrm{P}$, Lahart $\mathrm{C}$, Rosenblate R. The dimensions of perfectionism. Cogn Ther Res 1990; 14: 449-68

33 Baylé FJ, Bourdel MC, Caci H, Gorwood P, Chignon JM, Adés J, et al. Factor analysis of French translation of the Barratt Impulsivity Scale (BIS-10) [in French]. Can J Psychiatry 2000; 45: 156-65.

34 Patton JH, Stanford MS, Barratt ES. Factor structure of the Barratt Impulsivity Scale. J Clin Psychol 1995; 51: 768-74.

35 Bouvard $M$, Robbe Grillet $P$, Pham S, Milliery M, Amireche S, Fanget $F$, et al. Étude de validation d'une echelle multidimensionnelle de perfectionnisme [Validation study of a multidimensional perfectionism scale]. La Revue Francophone de Clinique Comportementale et Cognitive 2000; 5: 11-22.

36 Sanavio E. Obsessions and compulsions: the Padua Inventory. Behav Res Ther 1988; 26: 167-77.

37 Freeston $\mathrm{MH}$, Ladouceur R, Letarte H, Rhéaume J, Gagnon F, Thibodeau N. Measurement of Obsessive-compulsive Symptoms with the Padua Inventory: Replication and Extension. Unpublished research report School of Psychology, Laval University, Québec, Québec, Canada, 1994.

38 van oppen P, Hoekstra RJ, Emmelkamp PMG. The structure of obsessive-compulsive symptoms. Behav Res Ther 1995; 33: 15-23.

39 Lawson JS, Marshall WL, McGrath P. The social self-esteem inventory. Educ Psychol Measurem 1979; 39: 803-11.

40 Gauthier J, Samson P, Turbide D. Adaptation française du Social Self-Esteem Inventory [The French version of the Social Self-Esteem Inventory]. Can J Behav Sci 1981; 13: 218-25.

41 Devilly GJ, Borkovec TD. Psychometric properties of the credibility/ expectancy questionnaire. J Behav Ther Exp Psychiatry 2000; 31: 73-86.

42 Keijsers GPJ, Schaap CPDR, Hoogduin CAL, Hoogsteyns B, de Kemp ECM Preliminary results of a new instrument to assess patient motivation for treatment in cognitive-behaviour therapy. Behav Cogn Psychother 1999; 27: $165-79$

43 Pallanti S, Hollander E, Bienstock C, Koran L, Leckman J, Marazziti D, et al. International Treatment Refractory OCD Consortium. Treatment non-response in OCD: methodological issues and operational definitions Int J Neuropsychopharmacol 2002; 5: 181-91.

44 Wright $\mathrm{A}$, Rickards $\mathrm{H}$, Cavanna AE. Impulse-control disorders in Gilles de la Tourette syndrome. J Neuropsychiatry Clin Neurosci 2012; 24: 16-27.

45 Eddy CM, Mitchell IJ, Beck SR, Cavanna AE, Rickards HE. Altered attribution of intention in Tourette's syndrome. J Neuropsychiatry Clin Neurosci 2010; 22: $348-51$.

46 Seligman AW. Echolalia: A Novel. Hope Press, 1991. 